\title{
Can the full range of paramedic skills improve survival from out of hospital cardiac arrests?
}

\author{
R G Mitchell, U M Guly, T H Rainer, C E Robertson
}

Department of

Accident and

Emergency Medicine, The Royal Infirmary of Edinburgh, Lauriston Place, Edinburgh R G Mitchell

$T$ H Rainer

C E Robertson

Department of Accident and Emergency Medicine, Dundee Royal Infirmary, Barrack Road, Dundee U M Guly

Correspondence to Dr R G Mitchell, Department of Accident and Emergency Medicine, The Royal Infirmary of Edinburgh, Lauriston Place, Edinburgh EH3 9YW.

Accepted for publication 7 May 1997

\begin{abstract}
Objective-To examine the effect of full implementation of advanced skills by ambulance personnel on the outcome from out of hospital cardiac arrest. Setting-Patients with cardiac arrest treated at the accident and emergency department of the Royal Infirmary of Edinburgh.

Methods-All cardiorespiratory arrests occurring in the community were studied over a one year period. For patients arresting before the arrival of an ambulance crew, outcome of 92 patients treated by emergency medical technicians equipped with defibrillators was compared with that of 155 treated by paramedic crews. The proportions of patients whose arrest was witnessed by lay persons and those that had bystander cardiopulmonary resuscitation (CPR) were similar in both groups.

Results-There was no difference in the presenting rhythm between the two groups. Eight of 92 patients $(8.7 \%)$ treated by technicians survived to discharge compared with eight of $155(5.2 \%)$ treated by paramedics (NS). Of those in ventricular fibrillation or pulseless ventricular tachycardia, eight of $43(18.6 \%)$ in the technician group and seven of $80(8.8 \%)$ in the paramedic group survived to hospital discharge (NS). For patients arresting in the presence of an ambulance crew, four of 13 patients treated by technicians compared with seven of 15 by paramedics survived to hospital discharge. Only two patients surviving to hospital discharge received drug treatment before the return of spontaneous circulation.

Conclusions-No improvement in survival was demonstrated with more advanced prehospital care.

( 7 Accid Emerg Med 1997;14:274-277)
\end{abstract}

Keywords: cardiac arrest; prehospital care; advanced life support

Out of hospital cardiac arrest is the leading cause of sudden death in the United Kingdom. It is recognised that the system of prehospital care can affect survival rates from out of hospital cardiac arrest but, although widely differing survival rates have been reported, there has been little work to show what factors within a prehospital care system are associated with improved outcome.

In an attempt to improve prehospital care, the ambulance service within Scotland has introduced advanced training of ambulance personnel. ${ }^{1}$ This has been carried out in a stepwise manner. In 1989 all front line ambulances were equipped with semiautomatic defibrillators. Paramedic training was then started. This initially involved the practical skills of tracheal intubation and intravenous cannulation. From May 1994 the administration of specific cardioactive drugs was introduced into the Edinburgh area. All paramedics, whether recently qualified or previously trained, were able to give adrenaline, atropine, and lignocaine. Drug dosage used was according to the European Resuscitation Council guidelines, with the route of administration being either intravenous or through an endotracheal tube. For ease of administration the drugs come in preprepared syringes.

The success of equipping emergency ambulance crews with semiautomatic defibrillators has been reported. ${ }^{2}$ Recent experience in Edinburgh showed that tracheal intubation and intravenous cannulation without the administration of cardioactive drugs did not improve survival rates following out of hospital cardiac arrest. ${ }^{3}$

In this study we examine the effect of the full implementation of advanced skills by ambulance personnel on the outcome from out of hospital cardiac arrest. We compared success rates achieved by paramedics equipped with semiautomatic defibrillators and trained in intubation and drug administration with those achieved by technicians equipped only with semiautomatic defibrillators and simple airway adjuncts. Endpoints included return of spontaneous circulation, admission to, and discharge from hospital.

\section{Methods}

All patients with cardiac arrest treated at the accident and emergency (A\&E) department of the Royal Infirmary of Edinburgh were studied prospectively from 1 June 1994 to 31 May 
Table 1 Presenting characteristics for arrests before arrival of ambulance personnel

\begin{tabular}{lccl}
\hline & $\begin{array}{l}\text { Paramedics } \\
(n=155)\end{array}$ & $\begin{array}{l}\text { Technicians } \\
(n=92)\end{array}$ & P Value \\
\hline VF & $80(51.6 \%)$ & $41(46.7 \%)$ & NS \\
Asystole & $57(36.8 \%)$ & $47(46.7 \%)$ & NS \\
EMD & $18(11.6 \%)$ & $6(6.5 \%)$ & NS \\
Witnessed & $115(74.2 \%)$ & $69(75 \%)$ & NS \\
Bystander CPR & $57(36.8 \%)$ & $41(40.2 \%)$ & NS \\
\hline
\end{tabular}

$\mathrm{VF}$, ventricular fibrillation; EMD, electromechanical dissociation; CPR, cardiopulmonary resuscitation.

Table 2 Outcome for all presenting rhythms for arrests before arrival of ambulance crew

\begin{tabular}{lccl}
\hline & $\begin{array}{l}\text { Paramedics } \\
(n=155)\end{array}$ & $\begin{array}{l}\text { Technicians } \\
(n=92)\end{array}$ & $P$ Value \\
\hline ROSC & $44(28.4 \%)$ & $28(30.4 \%)$ & NS \\
Admitted & $33(21.3 \%)$ & $25(27.2 \%)$ & NS \\
Discharged & $8(5.2 \%)$ & $8(8.7 \%)$ & NS \\
\hline
\end{tabular}

ROSC, return of spontaneous circulation.

Table 3 Outcome for patients presenting in VF/Pulseless VT

\begin{tabular}{lcrl}
\hline & $\begin{array}{l}\text { Paramedics } \\
(n=80)\end{array}$ & $\begin{array}{l}\text { Technicians } \\
(n=43)\end{array}$ & $P$ Value \\
\hline ROSC & $32(40 \%)$ & $23(53.5 \%)$ & NS \\
Admitted & $24(30 \%)$ & $20(46.7 \%)$ & NS \\
Discharged & $7(8.8 \%)$ & $8(16.6 \%)$ & NS \\
\hline
\end{tabular}

ROSC, return of spontaneous circulation.

1995. Arrests from non-cardiac causes such as drug overdose or trauma, and those occurring in children under the age of 14 years or pregnant women, were excluded from further analysis. Arrests occurring in the accident and emergency department, and those patients receiving prehospital treatment by a hospital based physician-led team, Medic 1, were excluded. Arrests that were witnessed by ambulance personnel are analysed separately.

Paramedics attend a cardiorespiratory arrest either as part of the primary response or following a request from the on scene crew. This can either be for additional help with resuscitation or to assist in moving the patient. Selection of the first responding crew is carried out by ambulance control independently of this study. Their decision on crew allocation is based on the available crews and their proximity to the collapse. Approximately two thirds of crews during the study period were staffed by paramedics.

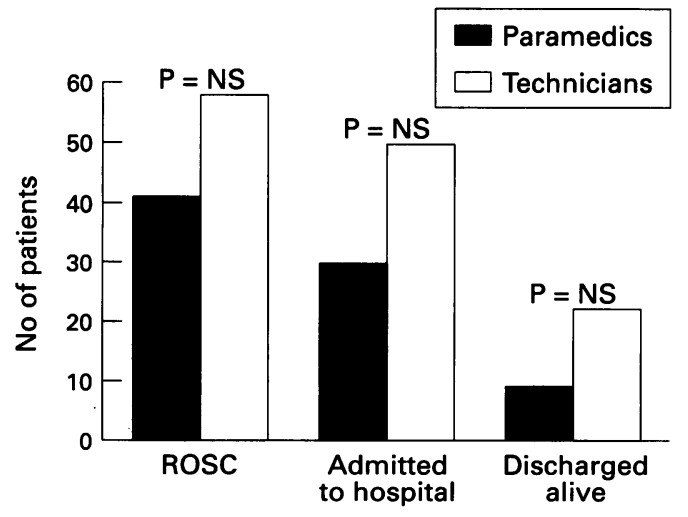

Figure 1 Percentage survival from witnessed VF/VT arrest occurring before the arrival of an ambulance crew.
The timing of prehospital events and interventions was taken from the Scottish Ambulance Service report forms and confirmed by the attending ambulance personnel. These include time of 999 call, time of ambulance arrival at and departure from the scene, and time of arrival at the $A \& E$ department. The presenting rhythm was recorded by the onscene crew and confirmed from the printout of the semiautomatic defibrillator (Laerdal Heartstart 2000 and 3000). Events recorded at the scene include the time of the first attempted defibrillation and the number of shocks given, whether tracheal intubation and intravenous cannulation were performed, and the administration of any drugs before arrival in the $A \& E$ department.

On arrival in the A\&E department, the ECG rhythm, any return of spontaneous cardiac output, and survival to hospital admission were documented. Discharge from hospital was recorded from inpatient hospital records.

Data were collected on a standard proforma based on the Utstein template ${ }^{4}$ and entered into a Database on Excel 4.0. Analysis was performed using a $\chi^{2}$ test with Yates correction for categorical variables. Continous variables were compared using a Mann-Whitney test for nonparametric data. A $P$ value $<0.05$ is taken as significant.

\section{Results}

Over the one year study period 409 patients were treated in the A\&E department following cardiac arrest: 275 fulfilled the entry criteria being of cardiac aetiology and occurring out of hospital; 247 of these occurred before the arrival of the ambulance crew and 28 patients arrested in the presence of ambulance personnel.

ARRESTS OCCURRING BEFORE THE ARRIVAL OF AMBULANCE CREW

Ninety two patients were treated by ambulance technicians (emergency medical technicians, EMT-Ds) and 155 by a paramedic ambulance crew. There are no demographic differences in the two groups. The mean age of patients treated by technicians was 67 years (range 29 to 90$)$ and $71(77.2 \%)$ were male. For the paramedic group the mean age was 69 years (range 16 to 93$)$, with $108(69.7 \%)$ male. In both groups a similar proportion of collapses was witnessed, $75 \%$ and $74.2 \%$, and received bystander cardiopulmonary resuscitation (CPR): $40.2 \%$ and $36.8 \%$ for technician and paramedic treated groups respectively. A greater proportion of arrests in the paramedic group had ventricular fibrillation/pulseless ventricular tachycardia (VF/pulseless VT) as the presenting rhythm $(51.6 \% v 46.7 \%)$ but this did not reach statistical significance (table 1 ).

For all presenting rhythms, eight of 92 patients $(8.7 \%)$ treated by technicians survived to hospital discharge, compared with eight of $155(5.2 \%)$ for paramedics (NS) (table 2). For those with a presenting rhythm of $\mathrm{VF} /$ pulseless VT, seven of 80 patients $(8.8 \%)$ treated by paramedics were discharged, compared with eight of $43(18.6 \%)$ treated by technicians (NS) (table 3). For patients with witnessed 
Table 4 Resuscitation time intervals

\begin{tabular}{llll}
\hline & Paramedics & Technicians & $P$ Values \\
\hline Call-response interval & $00: 07$ & $00: 08$ & NS \\
At scene interval & $00: 27$ & $00: 16$ & $<0.0001$ \\
Transport interval & $00: 08$ & $00: 10$ & $<0.004$ \\
$\begin{array}{l}\text { Call-arrival at A\&E } \\
\quad \text { interval }\end{array}$ & $00: 44$ & $00: 36$ & $<0.0001$ \\
\hline
\end{tabular}

Table 5 Outcome correlating with proceedure for arrests before arrival of ambulance personnel

\begin{tabular}{lcc}
\hline Proceedure & $\begin{array}{c}\text { No of } \\
\text { patients }\end{array}$ & $\begin{array}{c}\text { No } \\
\text { discharged }\end{array}$ \\
\hline Intravenous alone & 2 & 0 \\
Intubation alone & 20 & 1 \\
Intubation+intravenous access & 125 & $6^{\star}$ \\
Nil & 5 & 1 \\
Not recorded & 3 & 0 \\
\hline
\end{tabular}

* One patient received intravenous cardioactive drugs from his GP, a second patient received intravenous lignocaine after successful defibrillation.

collapse who were in VF/VT on arrival of the ambulance crew, eight of $38(21.1 \%)$ treated by technicians were discharged from hospital, compared with seven of $73(9.6 \%)$ treated by paramedics $(0.1>\mathrm{P}>0.05)$ (fig 1$)$.

For presenting rhythms for which a shock was not indicated, 49 patients were treated by technicians with no survivor to hospital discharge. Seventy five were treated by paramedics, of whom one survived. This patient received full advanced life support techniques before return of spontaneous circulation.

There was no significant difference in the call-response intervals for patients treated by ambulance technicians and paramedics. The median call-response times was 00:08 minutes for EMT-Ds and 00:07 minutes for paramedics. EMT-Ds took longer in the transporting from the scene to the $\mathrm{A} \& \mathrm{E}$ department, with median intervals of 00:10 minutes for technicians and 00:08 minutes for paramedics $(P<$ $0.004)$. The time spent at the scene was longer for paramedics, with a median time of $00: 27$ minutes compared with 00:16 minutes for EMT-Ds $(P<0.001)$. This resulted in a significant increase in the length of time from the 999 call to the patient arriving at the $A \& E$ department for patients treated by paramedics. The median call to hospital interval was 00:44 minutes for paramedic treated patients and 00:36 minutes for those treated by EMT-Ds ( $P$ $<<0.001$ ) (table 4).

Of the 155 patients treated by paramedics, 125 were intubated and had intravenous access obtained, 20 only had intubation performed, and two only had intravenous access performed. Five patients had neither procedure performed and in three cases inadequate records were made (table 5 ).

Eight patients treated by paramedics, who arrested before arrival of the ambulance crew, survived to hospital discharge. Five of these did not receive drugs from the attending paramedic crew. One patient only required defibrillation, three were treated by defibrillation and tracheal intubation, and one had drugs given by a general practitioner. Three patients who survived to hospital discharge received drugs given by the attending paramedic crew. The
Table 6 Outcome for all presenting rhythms for arrests witnessed by ambulance personnel

\begin{tabular}{llll}
\hline & $\begin{array}{l}\text { Paramedics } \\
(n=15)\end{array}$ & $\begin{array}{l}\text { Technicians } \\
(n=13)\end{array}$ & P Value \\
\hline ROSC & 9 & 10 & NS \\
Admitted & 7 & 8 & NS \\
Discharged & 7 & 4 & NS \\
\hline
\end{tabular}

ROSC, return of spontaneous circulation.

first patient was in ventricular fibrillation and regained spontaneous output after four dc shocks and $1 \mathrm{mg}$ of adrenaline intravenously. The second was in asystole and was given $1 \mathrm{mg}$ of adrenaline and $3 \mathrm{mg}$ of atropine intravenously. The third was defibrillated from ventricular fibrillation to atrial fibrillation and received $100 \mathrm{mg}$ of lignocaine intravenously.

Four patients had drugs given at the scene by a general practitioner. Two patients were treated by paramedics, one of whom survived, and two were treated by EMT-Ds, neither of whom survived. All of these patients are included in the analysis.

ARRESTS WITNESSED BY THE AMBULANCE CREW Twenty eight patients arrested in the presence of ambulance personnel, of whom 15 were treated by paramedics and 13 by technicians. In each group seven patients entered a shockable rhythm as their first presenting rhythm; six of seven patients treated by paramedics and four of seven treated by technicians survived to hospital discharge, but this does not reach statistical significance. Of those patients who were discharged, only one received any drug treatment $-100 \mathrm{mg}$ lignocaine intravenously after return of spontaneous circulation. Of the 14 patients arresting with a rhythm for which a shock was not indicated, only one survived to hospital discharge. He was treated by paramedics, having a return of spontaneous circulation with external cardiac massage and bag-valve mask respiration alone (table 6).

\section{Discussion}

The effect of a mobile intensive care unit on survival from acute myocardial infarction and out of hospital cardiac arrest was described by Pantridge and Geddes in $1967,{ }^{5}$ but the optimal management of out of hospital cardiac arrest remains uncertain. The progression of prehospital care provision by the Scottish Ambulance Service gives an opportunity to access the relative contributions made by different interventions to the outcome from out of hospital cardiac arrest.

The impact of semiautomatic defibrillation in the Heart-Start Scotland campaign has been documented previously. ${ }^{26}$ Recent experience in Edinburgh of the impact on out of hospital cardiac arrest by paramedics using advanced airway skills and intravenous cannulation, without drug administration, did not show any improvement in outcome with these techniques. ${ }^{3}$

Not surprisingly, this study confirms that the presence of a paramedic at the scene significantly prolongs the "at-scene" time and the 
"call-to-hospital" interval. This may be at least in part because paramedics attend a patient following cardiac arrest as a first response, that is, as part of the first ambulance crew to arrive, or as a secondary response following initial response by an EMT-D crew. The wait for a secondary response to arrive would markedly prolong the at-scene interval. This increase in at-scene time and delay in the patient reaching hospital is not warranted when there is currently no evidence that the additional skills contributed by paramedics improve survival.

It was to be expected that merely siting an intravenous cannula without administration of any drugs would have no effect on outcome from cardiac arrest. ${ }^{3}$ In this study, however, we cannot show any improvement in overall survival with the full range of paramedic skills used in the United Kingdom, that is, tracheal intubation, intravenous cannulation, and cardioactive drug administration. For arrests occurring before the arrival of an ambulance crew, all eight survivors treated by EMT-Ds had a presenting rhythm of VF/VT and required three defibrillating shocks or less to return to a spontaneous perfusing rhythm, whereas seven of eight survivors treated by paramedics were in VF/VT all had return of spontaneous circulation with four shocks or less. For all patients sustaining a VF/VT arrest the median time interval from call to defibrillation for survivors was eight minutes and for non-survivors, 12 minutes. This further emphasises the importance of early defibrillation.

For those patients who had an arrest which was witnessed by ambulance personnel there was a trend towards an improved survival in the group treated by paramedics. However, for all presenting rhythms in this group no patient who survived to hospital discharge received skills other than defibrillation and basic life support.

To date no study has shown improved survival to hospital discharge from the admin- istration of any cardioactive drugs. This study confirms these findings, with only four survivors having had drugs given by paramedics. Two patients had been defibrillated into rhythm with an output and received lignocaine. A third was defibrillated to spontaneous output with four shocks and, as recommended in the European resuscitation guidelines, ${ }^{7}$ received $1 \mathrm{mg}$ adrenaline. The fourth responded rapidly to CPR, $1 \mathrm{mg}$ adrenaline, and $3 \mathrm{mg}$ atropine.

CONCLUSION

In this study we were unable to demonstrate any objective benefit from the use of full paramedic skills, that is, tracheal intubation, intravenous cannulation, and drug administration, in the management of out of hospital cardiac arrest. The important and proven factors that lead to outcome improvements remain basic life support and rapid defibrillation. ${ }^{8}$

1 National Health Service Training Directorate. National Health Service training manual. Bristol: NHSTD, Swindon Press, 1991.

2 Cobbe SM, Redmond MJ, Watson JM, Hollingworth J, Carrington DJ. Heartstart Scotland-initial experience of a national service for out of hospital fibrillation. BMF 1991; national service

3 Guly UM, Mitchell RG, Cook R, Steedman DJ, Robertson CE. Paramedics and technicians are equally successful at managing cardiac arrest outside hospital. $B M \mathcal{F} 1995 ; 310$ : $1091-4$

4 Cummins RO, Chamberlain DA, Abramson NS, Allen M, Baskett P, Becker L, et al. Recommended guidelines for uniform reporting of data from out of hospital cardiac arrest: the Utstein style. Resuscitation 1991;21;960-75.

5 Pantridge JF, Geddes JS. A mobile intensive care unit in the management of myocardial infarction. Lancet 1967;ii: manage

6 Sedgewick ML, Watson JM, Dalziel K, Carrington DJ, Cobbe SM. Efficacy of out of hospital defibrillation by ambulance technicians using automated external defibrillators. The Heartstart Scotland project. Resuscitation 1992; 24:73-87.

7 Chamberlain DA, Bossart L, Carli P, Edgren E, Ekstrom L, Hapnes S, et al. (ALS working party of the European Resuscitation Council.) Guidelines for advanced life Resuscitation Council.) Guidelines for
support. Resuscitation 1992;24:111-21.

8 Cummins RO, Ornato JP, Thies WH, Pepe PE. Improving survival from sudden cardiac arrest: the chain of survival concept. Circulation 1991;83:1832-47. 